\title{
Two-Dimensional Forward-Backward Time-Stepping Approach for Tumor Detection in Dispersive Breast Tissues
}

\author{
Kismet Anak Hong Ping ${ }^{1} \quad$ Toshifumi Moriyama $^{2} \quad$ Takashi Takenaka $^{2} \quad$ Toshiyuki Tanaka $^{1}$ \\ ${ }^{1}$ Graduate School of Science and Technology, \\ ${ }^{2}$ Department of Electrical and Electronic Engineering, \\ Nagasaki University, 1-14 Bunkyo-machi, Nagasaki 852-8521, JAPAN. \\ hpkismet@yahoo.com or takenaka@net.nagasaki-u.ac.jp
}

\begin{abstract}
The Forward-Backward Time-Stepping (FBTS) technique is applied to determine the presence and location of malignant tumor in the heterogeneous breast model. A new strategy is integrated in the FBTS algorithm to accurately estimate the dielectric properties of tissues. We demonstrate 2-D FBTS technique utilizing the numerical dispersive breast model in a free space. This new strategy manipulating Debye dispersion equation is proposed to treat dispersive case. Numerical simulation results show the FBTS algorithm has the potential to provide useful quantitative information of the breast's internal composition.
\end{abstract}

Keywords-Breast Cancer Detection; Inverse Scattering; Debye dispersion; Dispersive; FBTS.

\section{INTRODUCTION}

Breast cancer still remains the leading cause of cancer death among women [1]. Early detection is an important issue for effective treatments. Currently, X-ray mammography is the standard method of breast screening for detecting tumors. Limitations of X-ray mammography have been shown to miss up to $15 \%$ of tumors [2] and high false reading rates (see [3] and references therein).

Recently, microwave imaging for breast cancer detection has been reported by many researchers [3]-[5] as alternatives to $\mathrm{X}$-ray mammography. Electrical properties of tissues at microwave frequencies have been extensively studied [6], [7]. There are varieties of active microwave approaches utilizing frequency-domain inverse scattering [8], and ultra-wideband radar-based techniques [9] for breast imaging. These approaches have demonstrated the ability for detecting tumors both in numerical studies and phantom experiments.

In this paper, we present the Forward-Backward TimeStepping (FBTS) technique using broadband microwave signals to solve the inverse scattering problem in the time domain. This technique was reported by Takenaka et. al. [10] for a simple one-dimensional case and has been extended to reconstruction of a heterogeneous breast model in twodimensional [11], [12]. For three-dimensional case, both numerical and experimental have been studied [13], [14]. The use of coupling liquid as the background medium reported in [11] demonstrated the efficacy of two-dimensional (2-D) FBTS technique for tumors detection and quantitative analysis of the breast's internal composition.

In this study, we focus the research work of 2-D FBTS utilizing the numerical dispersive breast model in a free space. The main goal in this study is to accurately estimate the size, shape and dielectric properties of the malignant tumor and healthy breast tissues for dispersive case. We will assume that the breast model is composed of four different tissue types with distinct dielectric properties: skin, fatty, fibroglandular and tumor. In order to achieve this goal, we exploit the single-pole Debye dispersion equation to be incorporated directly into FBTS algorithm.

\section{METHODS}

\section{A. Dispersive Dielectric Properties Estimation}

The foundation of this approach lays in the formulation of the Debye dispersion equation. It uses an approximate relation between the relative permittivity and conductivity. Even though there is no such relation, however, if we can approximate a nonlinear relationship explicitly, the relationship is used to reconstruct only a relative permittivity profile. The frequency dependence of the dielectric properties of breast tissue is modeled using a single-pole Debye dispersion equation for the complex relative permittivity

$$
\varepsilon_{r}^{*}(\omega)=\varepsilon_{\infty}+\frac{\varepsilon_{s}-\varepsilon_{\infty}}{1+j \omega \tau}-j \frac{\sigma_{s}}{\omega \varepsilon_{0}}
$$

where $\varepsilon_{\infty}$ is the relative permittivity at infinite frequency, $\varepsilon_{s}$ is the static relative permittivity, $\tau$ is the relaxation time, and $\sigma_{s}$ is the static conductivity.

In this framework, we derive equation (1) to be expressed as

$$
\varepsilon_{r}^{*}(\omega)=\left\{\varepsilon_{\infty}+\frac{\varepsilon_{s}-\varepsilon_{\infty}}{1+(j \omega \tau)^{2}}\right\}-j\left\{\frac{\omega \tau\left(\varepsilon_{s}-\varepsilon_{\infty}\right)}{1+(j \omega \tau)^{2}}+\frac{\sigma_{s}}{\omega \varepsilon_{0}}\right\}
$$

The first term of expression in equation (2) can be represented as relative permittivity given by 


$$
\varepsilon_{r}(\omega)=\varepsilon_{\infty}+\frac{\varepsilon_{s}-\varepsilon_{\infty}}{1+(j \omega \tau)^{2}}
$$

Take into consideration of equation (3) and second term of expression in equation (2), the conductivity is then expressed as

$$
\sigma(\omega)=\omega^{2} \tau \varepsilon_{0} \varepsilon_{r}(\omega)+\left(\sigma_{s}-\omega^{2} \tau \varepsilon_{0} \varepsilon_{\infty}\right)
$$

\section{B. FBTS Inverse Scattering Technique}

In the FBTS technique, errors between measured and calculated microwave scattering data are compared in the time domain. The following is the error functional equation for an assumed electrical parameter vector, $\boldsymbol{p}$ consists of permittivity and conductivity.

$$
Q(\mathbf{p})=\int_{0}^{T} \sum_{m=1}^{M} \sum_{n=1}^{N}\left|v_{m}\left(\mathbf{p} ; \mathbf{r}_{n}, t\right)-\widetilde{v}_{m}\left(\mathbf{r}_{n}, t\right)\right|^{2} d t
$$

where $\widetilde{v}_{m}\left(\boldsymbol{r}_{n}, t\right)$ and $v_{m}\left(\boldsymbol{p} ; \boldsymbol{r}_{n}, t\right)$ are the measured electric field in time domain at the receiving position $n$ due to a pulse radiated by a transmitter $m$ and the corresponding calculated electric field for an estimated electric parameters $\boldsymbol{p}$, respectively. The gradient of the error functional with respect to $\boldsymbol{p}$ can be calculated utilizing a forward Finite-Difference Time-Domain (FDTD) computation followed by a corresponding adjoint FDTD computation in which residual received signals denoted as $\left[v_{m}\left(\boldsymbol{p} ; \boldsymbol{r}_{n}, t\right)-\widetilde{v}_{m}\left(\boldsymbol{r}_{n}, t\right)\right]$ are utilized as equivalent sources which are reversed in time. In this approach, we use Polak-Ribiète-Polyak conjugate gradient method for optimization technique to solve the inverse scattering problem. Refer to [10] for more details.

\section{2-D MRI-derived Breast Model}

A slice of MRI contains $0.39 \mathrm{~mm} \times 0.39 \mathrm{~mm}$ resolution from the healthy left breast of a volunteer in the prone position was taken at Nagasaki University Hospital. First order Debye model parameters were mapped to each pixel value using the piecewise-linear procedure described in [15]. The model was resized to a $1.0 \mathrm{~mm} \times 1.0 \mathrm{~mm}$ resolution to satisfy the FDTD grid size. The skin layer of $2.0 \mathrm{~mm}$ thickness was added to the model. Then, a $5.0 \mathrm{~mm}$ diameter tumor was added in the fibroglandular region of the model. The values of the nominal Debye parameters for the various tissues were taken from [15].

\section{Image Reconstructions}

To demonstrate the validity of the FBTS technique, we consider reconstruction of a 2-D numerical dispersive breast model. 2-D FDTD in $T M_{z}$ wave numerical simulations are carried out to obtain synthetic scattering data as measured scattering data. Sixteen points represented as antennas encircles the breast as shown in Figure 1. Each of the 16 points was used sequentially as a transmitter represented by a simple line source in the FDTD simulation with the electric fields being sampled at the remaining 15 points. This set of scattering calculations representing 240 transmitter/receiver combinations formed a set of measured data denoted as $\widetilde{v}_{m}\left(\boldsymbol{r}_{n}, t\right)$ term of equation (5).
A sinusoidally modulated Gaussian pulse was used for excitation signal having bandwidth of $1.3 \mathrm{GHz}$ centered at $2.0 \mathrm{GHz}$.

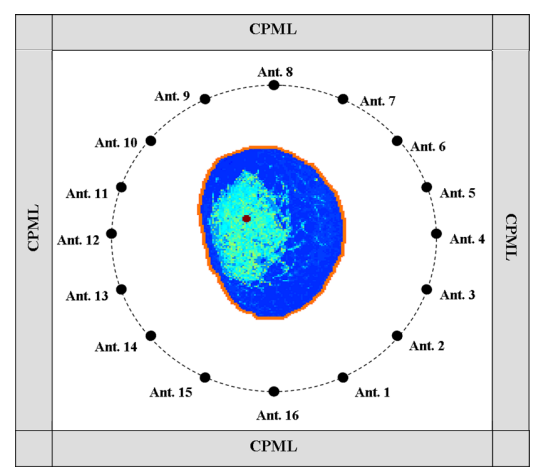

Figure 1. Simulation Setup

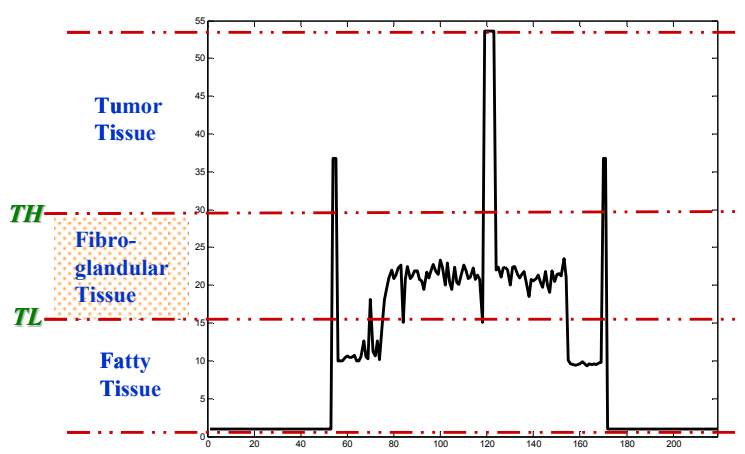

Figure 2. Level Condition

In this study, the breast was assumed to be immersed in a free space as a background medium. After forming the set of synthetic scattering data, the FBTS technique was utilized to reconstruct the breast model. Reconstructions were conducted utilizing a $1.0 \mathrm{~mm} \times 1.0 \mathrm{~mm}$ FDTD grid size and the optimization was carried out for 150 iterations. The Convolution Perfectly Matched Layer (CPML) consisting of fifteen-cell size is used for the FDTD solution space. Next, we consider the effect of a priori information to estimate the electrical parameters of the tumor in the breast model on the convergence behavior of the FBTS algorithm. The crucial feature in this approach is to determine the two possible values (denoted as $T L$ and $T H$ ) describing the minimum and maximum levels of the fibroglandular tissue region as illustrated in Figure 2. Assume that the electric properties of skin layer are known. It is assumed that these two values are known as priori information of relative permittivity level condition for fibroglandular tissue. The outline of reconstructions is chosen as follows:

- Estimate A: A tumor of 5.0mm diameter was added in the fibroglandular region of the model. The reconstruction region matches the shape of the breast. The initial guess $\varepsilon_{r}$ and $\sigma$ values in the entire breast region are set equal to the average value of "normal" breast region ( $\varepsilon_{r}=13.9$ and $\sigma=0.27$ ) which includes the 
fat, fibroglandular, and transition regions. Then, we apply $T L=15$ and $T H=30$ to the level condition.

- Estimate B: Similar setup as in Estimate $\boldsymbol{A}$. Then, we consider the level condition value for $T H=35$ whereby the value of $T L$ remains the same as in Estimate $\boldsymbol{A}$.

\section{RESUlts AND DiscUSSION}

From a mathematical viewpoint, the relative permittivity $\varepsilon_{r}$ and conductivity $\sigma$ at a fixed frequency seems to have a linear relation. In reality, this relation is invalid. However, in this approach we assume that they have a linear relation to each other. When $\varepsilon_{r}$ changes, $\varepsilon_{\infty}, \varepsilon_{s}$ and $\sigma_{s}$ (assume $\tau$ is remain unchanged) also change so that $\sigma$ does not change linearly with $\varepsilon_{r}$. However, for simplicity, we assume that the second term of equation (4) keep constant given by nominal values of $\varepsilon_{\infty}$ and $\sigma_{s}$. As a result, conductivity $\sigma$ can be obtained. Finally, modification on the gradient of a cost functional with respect to the relative permittivity is considered in order to take account of the effect of conductivity. Obtaining this relation, on the other hand, could lead to new strategy in FBTS technique to reconstruct the conductivity profile based on reconstructed relative permittivity from dispersive dataset of the 2-D MRIderived breast model.

Figure 3 and 4 show the reconstructed relative permittivity and conductivity of the Estimate $\boldsymbol{A}$ and Estimate $\boldsymbol{B}$, respectively. From these figures, it is found that the tumor is clearly detected and the shape of the fibroglandular region is distinct from the surrounding fat tissue. This approach can be considered to treat dispersive breast model. The level condition (TL and $T H$ ) would influence the shape of reconstructed conductivity. This level condition is a good approximation for describing the minimum and maximum values of the fibroglandular tissue between fatty tissue and tumor tissue. In this study, the 2-D FBTS algorithm was performed on a cluster of 16 Personal Computers (PCs) and parallel processing integrated with Message Passing Interface (MPI). Each computer has a CPU of Intel ${ }^{\circledR}$ Core $^{\mathrm{TM}} 2$ QUAD Q9450 $2.66 \mathrm{GHz}$ and $8 \mathrm{~GB}$ memory.

\section{CONCLUSION}

The FBTS algorithm is an iterative technique in time domain to solve the inverse scattering problem of reconstructing relative permittivity and conductivity profiles. The FBTS technique was used to successfully detect malignancy embedded in a dispersive breast model. The numerical simulation results in this study indicate that the new approach in the FBTS algorithm accurately estimate the shape and location of a tumor in order to discriminate between malignant and healthy breast tissue, and also can assess to the breast's internal composition.

\section{ACKNOWLEDGMENT}

This work was partially supported by a Japan Society for the Promotion of Science (JSPS) Grant-in-Aid for Scientific Research (B) (no. 20360187). The authors would like to express their thanks to Dr. Jessi Ernest Johnson for his assistance in providing the numerical data of the MRI-derived breast model. He was a Postdoctoral Fellow in Department of Electrical and Electronic Engineering at Nagasaki University, Japan.

\section{REFERENCES}

[1] American Cancer Society, Inc., Cancer Facts \& Figures 2007. American Cancer Society, 2007

[2] E.C. Fear, S.C. Hagness, P.M. Meaney, M. Okoniewski and M.A. Stuchly, "Near-field imaging for breast tumor detection", IEEE Microwave Magazine, vol. 3, Mar. 2002, pp 48-56.

[3] E.C. Fear, X. Li, S.C. Hagness, M.A. Stuchly, "Confocal Microwave Imaging for Breast Cancer Detection: Localization of Tumors in Three Dimensions," IEEE Trans. Biomed. Eng., vol. 49, no. 8, 2002, pp. 812821.

[4] J. Sill and E. Fear, "Tissue senseing adaptive for breast cancer detection - experimental investigation of simple tumor models," IEEE Trans. Microw. Theory Tech., vol. 53, 2005, pp. 3312-3319.

[5] E. Bond, X. Li, S. Hagness, and B. Van Veen, "Microwave imaging via space-time beamforming for early breast cancer," IEEE Trans. Antennas Propag., vol. 51, no.8, Aug 2003, pp. 1690-1705.

[6] A. Rosen, M. A. Stuchly, and A. Vander Vorst, "Applications of RF/Microwaves in Medicine," IEEE Trans. Microwave Theory Tech. 50, 2002, pp. 963-974.

[7] M. Lazebnik, D. Popovic, L. McCartney, C. B.Watkins, M. J. Lindstrom J. Harter, S. Sewall, T. Ogilvie, A. Magliocco, T. M. Breslin, W. Temple, D. Mew, J. H. Booske, M. Okoniewski, and S. C. Hagness, "A largescale study of the ultrawideband microwave dielectric properties of normal, benign and malignant breast tissues obtained from cancer surgeries," Physics in Medicine and Biology, vol. 52, no. 20, 2007, pp. 6093-6115.

[8] F. Qianqian, P. Meaney, S. Geimer, A. Streltsov, and K. Paaulsen, "Microwave image reconstruction from 3-d fields coupled to 2-d parameter estimation,” IEEE Trans. Med. Imag., vol. 23, no. 4, 2004, pp. 475-484.

[9] X. Li, S. Davis, S. Hagness, D. Van Der Weide, and B. Van Veen, "Microwave imaging via space-time beamforming: experimental investigation of tumor detection in multilayer breast phantoms," IEEE Trans. Microw. Theory Tech., vol. 52, no. 8, Aug. 2004, pp. 1856-1865.

[10] T. Takenaka, H. Jia, and T. Tanaka, "Microwave imaging of electrical property distributions by a forward-backward time-stepping method," J. Electromagn. Waves Applicat., vol. 14, no. 12, 2000, pp. 1609-1626.

[11] J.E. Johnson, T. Takenaka and T. Tanaka, "Two-dimensional Timedomain Inverse Scattering for Quantitative Analysis of Breast Composition”, IEEE Trans. Biomed. Eng. , vol. 55, no. 8, 2008, pp. 1941-1945

[12] K. A. H. Ping, T. Moriyama, T. Takenaka and T. Tanaka, "Reconstruction of breast composition in a free space utlizing 2-D forward-backward time-stepping for breast cancer detection," Advances in Medical, Signal and Information Processing 2008 (MEDSIP 2008), $4^{\text {th }}$ IET Internatioal Conf., 2008, pp.1-4.

[13] J. Johnson, H. Zhou, and T. Takenaka, "Experimental three-dimensional time-domain reconstruction of dielectric objects for breast cancer detection," Mediterranean Microwave Symposium, 2006, pp. 423-426.

[14] T. Moriyama, Y. Yamaguchi, K. A. Hong Ping, T. Tanaka, and T. Takenaka, "Parallel Processing of Forward-Backward Time-Stepping Method for Time Domain Inverse Scattering," PIERS ONLINE, Vol. 4, No. 6, 2008

[15] M. Converse, E. Bond, B. Veen and S. Hagness, “A computational study of ultra-wideband versus narrowband microwave hyperthermia for breast cancer treatment," IEEE Trans. Microw. Theory Tech., vol. 54, no. 5, May 2006, pp. 2160-2180. 


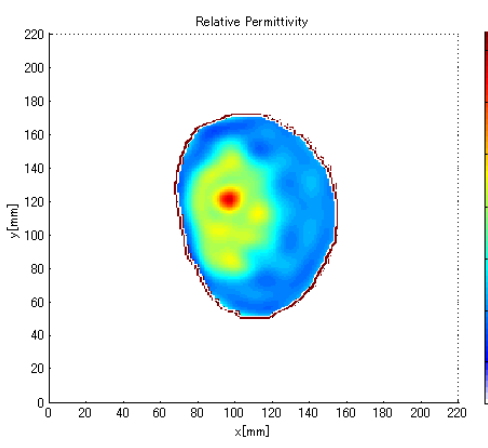

(a) Relative permittivity reconstruction

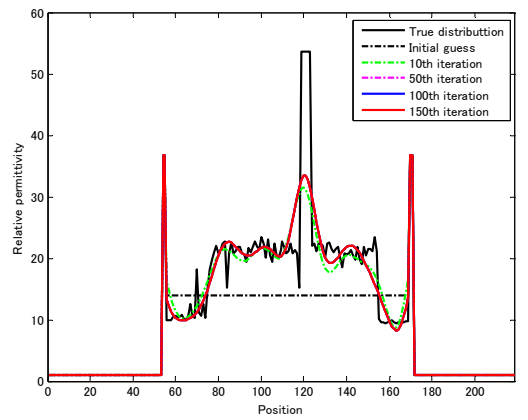

(c) Relative permittivity $(\mathrm{x}=96 \mathrm{~mm})$

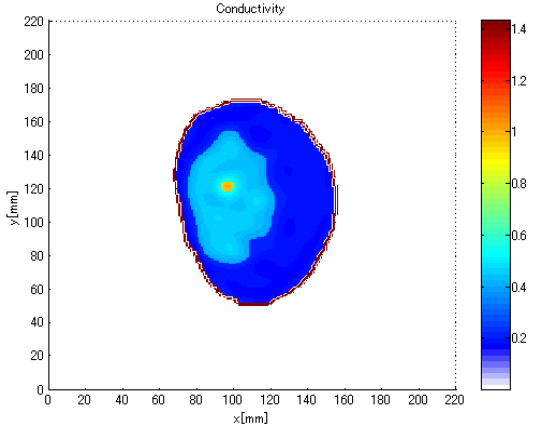

(b) Conductivity reconstruction

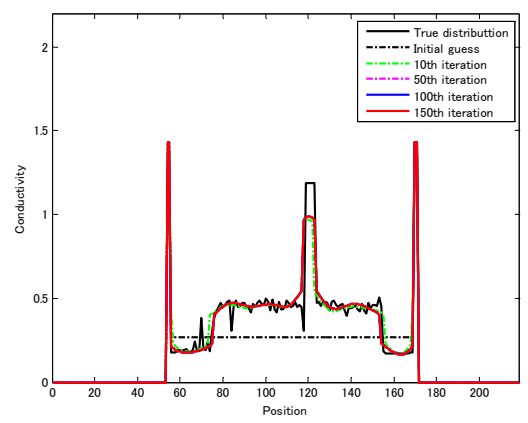

(d) Conductivity $(\mathrm{x}=96 \mathrm{~mm})$

Figure 3. Results of Estimate A

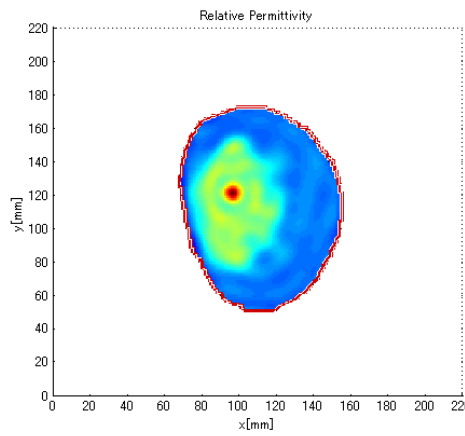

(a) Relative permittivity reconstruction

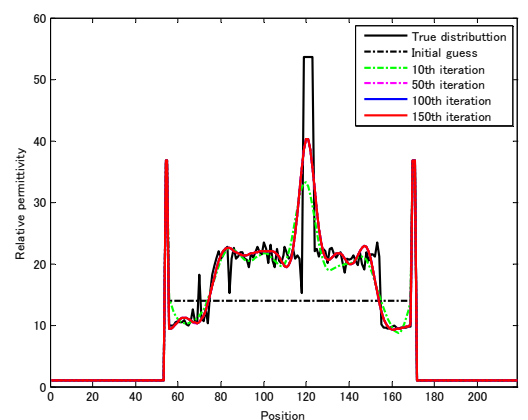

(c) Relative permittivity $(\mathrm{x}=96 \mathrm{~mm})$

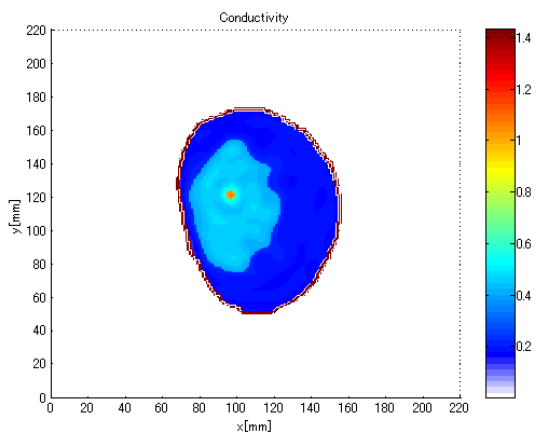

(b) Conductivity reconstruction

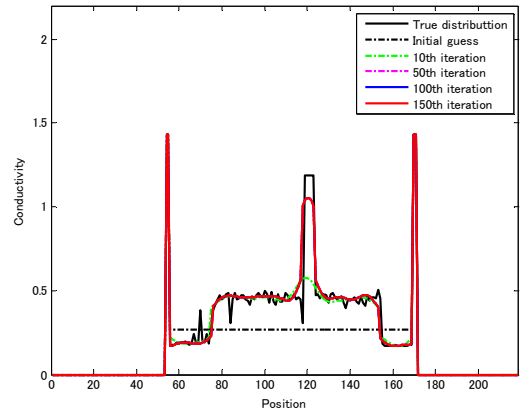

(d) Conductivity $(\mathrm{x}=96 \mathrm{~mm})$

Figure 4. Results of Estimate B 